\title{
SIMULATION OF RADIATIVE HEAT FLUX DISTRIBUTION UNDER AN INFRARED HEAT EMITTER
}

\author{
JAN LOUfEK*, JiŘina KRÁlovCová \\ Technical University of Liberec, Faculty of Mechatronics, Informatics and Interdisciplinary Studies, Studentská \\ 1402/2, 561 17, Liberec, Czech Republic \\ * corresponding author: jan.loufek@tul.cz
}

\begin{abstract}
This paper deals with a heat radiation model used forcalculating the heat flux of an infrared heater. In general, we consider a system consisting of a set of objects, whereby a single object can stand for a heater, a reflector or a heated body. Each of the objects is defined by its bounding surface. The model applies a 2D restriction of the real system. The aim of a particular simulation is to obtain the heat flux distribution all over the heated body under given conditions, e.g. the temperature and material properties of the object. The implemented model is used to design a reflector profile to obtain a desired heat flux distribution. The paper presents the implemented model, a validation of simulation using measured data, and an example of the design of a reflector.
\end{abstract}

KEYWORDS: radiative heat transfer; simulations; optimization; heat flux distribution.

\section{INTRODUCTION}

In high temperature applications, e.g. with infrared heating, thermal radiation is the dominant mode of heat transfer. Infrared heating is used, among other methods, to heat-up shell-forms in the production of artificial leathers in the automotive industry. In this case, it is necessary to determine the optimal location for heaters, aiming at almost uniform distribution for radiation intensity all over the shell form surface. The procedure for determining the optimal location of particular heaters is based on temperature simulation and optimization. Some relevant issues were published in [1]3. A similar topic is adresses Rukolaine [4, whose work presents the possibility of optimal shape design related to radiative heat transfer. In [4] work the inverse problem is reduced by a least squares objective functional to an optimised one. In our work we consider a reflector of gray body properties, i.e. the case when the energy incident on a surface is reflected diffusely.

A model of a complex system consisting of a shell form and dozens (or hundred) of heaters [5] works with a heat flux distribution function that determines the heat flux at various points under the emitter. Unfortunately, there are not enough data specifications available for an infrared heater that would allow an evaluation of the necessary characteristics. The data needed can be achieved by measurements of the heat flux coming out of the heater at different distances and different incidence angles. Furthermore, it is necessary to make an interpolation to find contribution of a particular heater to the heat flux at a respective point of the shell form. Another method for obtaining the heat flux distribution under particular heaters is to simulate them. For that purpose we implemented a model of heat transfer by radiation, where the system consists of a heat emitter, a reflector and a heated body. A similar topic, i.e., radiative heat transfer simulation, can be found in the work of Takami, Danielson and Mahmoudi [6], where the authors present simulations for the purpose of optimizing the high power reflector with respect to heat power and temperature distribution.

Our model is also applicable to other purposes: (1), to verify a multiple infrared heat source interaction, or (2) to design an alternative reflector shape suitable for specific criteria, such as focusing the heat flux in the desired direction or (3) to design a reflector that achieves a desired heat flux distribution all over the heated body.

\section{HEAT TRANSFER MODEL}

The following section summarizes the general relations used in the model design. The model presented here works with two main parts of the system. There is a heat source or a set of heat sources that is/are equipped with reflectors (if needed) on one side and the object or set of objects to be heated up on the other side. In general, each part of the system radiates a continuous electromagnetic energy. The magnitude of the energy depends on the temperature of the object and its surface properties. The amount of radiated energy depends on the biquadrate of the absolute temperature. In the case of a "black body", i.e. a body, which absorbs/emits all the incident energy without any reflection, the amount of energy absorbed or emitted per square meter $e_{b}\left(\mathrm{~W} \mathrm{~m}^{-2}\right)$ is described by the Stefan-Boltzmann law

$$
e_{b}=\sigma T^{4}
$$

where $\mathrm{T}(\mathrm{K})$ stands for the absolute temperature and $\sigma=5.670373 \cdot 10^{-8} \mathrm{~W} \mathrm{~m}^{-2} \mathrm{~K}^{-4}$ is the StefanBoltzmann constant. 
In the case of a real body ("gray body"), we deal with emissivity of the body surface $\varepsilon(-)$, which represents the rate of emitted energy against the black body emission.

$$
\varepsilon=\frac{e(T)}{e_{b}(T)}=\frac{\int_{0}^{\infty} e_{\lambda}(\lambda, T) \mathrm{d} \lambda}{\sigma T^{4}}
$$

where $\lambda(m)$ stands for wavelength of radiating energy.

We assume that each part of the system (every heater, every reflector, every heated object) is determined (set) by its bounding surface. The union of all the bounding surfaces forms the model domain. For the purposes of radiation heat transfer simulation, we introduced a partition of the domain into a set of facets as simplex elements of the respective domain (i.e. line segments for the bounding surface in $2 \mathrm{D}$, and triangles for the bounding surface in 3D). The union of the facets forms an approximation of the original domain. Each facet is described by its position, its normal vector, its surface temperature and emissivity.

The key concept in solving the radiative heat transfer in systems of surfaces is demonstrated by the view factor. The view factor $F_{i-j}$ is defined as the fraction of the total outgoing radiation out of the $i$-th surface intercepted by the $j$-th surface. The view factor $F_{i-j}$ is demonstrated by the double-integral form

$$
F_{i-j}=\frac{1}{A_{i}} \int_{A_{i}} \int_{A_{j}} \frac{\cos \beta_{1} \cos \beta_{2}}{\pi s^{2}} \mathrm{~d} A_{i} \mathrm{~d} A_{j},
$$

where $A_{i}\left(\mathrm{~m}^{2}\right)$ stands for the area of the surface, $s(\mathrm{~m})$ stands for the length of the join of particular points on surfaces $A_{i}$ and $A_{j}$, and $\beta_{n}$ (rad) stands for the angle between the join and outer normal. A sample configuration of two rectangular facets in $3 \mathrm{D}$ is shown in Figure 1 .

For some simple geometries, calculations of the view factors are straightforward and various methods have been outlined in books on radiative heat transfer, e.g. in $[7-9]$. Due to the asymptotic complexity $O\left(n^{4}\right)$ of the algorithm, related to the numerical evaluation of the view factor, which is unavoidable for complex geometries, especially the geometry working with objects forming obstacles, the calculation is a time-consuming problem. It particularly approves simulations with a more accurate discretization, where the considered bounding surfaces are split into plenty of discrete facets.

The amount of surface density of the radiated energy (radiosity) $B\left(\mathrm{~W} \mathrm{~m}^{-2}\right)$ is determined as the sum of the reflected and the emitted energy, and is given by the formula

$$
B=\rho H+\varepsilon e_{b},
$$

where $H\left(\mathrm{~W} \mathrm{~m}^{-2}\right)$ stands for the irradiance of the surface and $\rho$ (dimensionless) stands for the reflectivity of the considered surface. Eventually, the rate of heat transfer $Q_{i}(\mathrm{~W})$ of particular surface $A_{i}$ in the

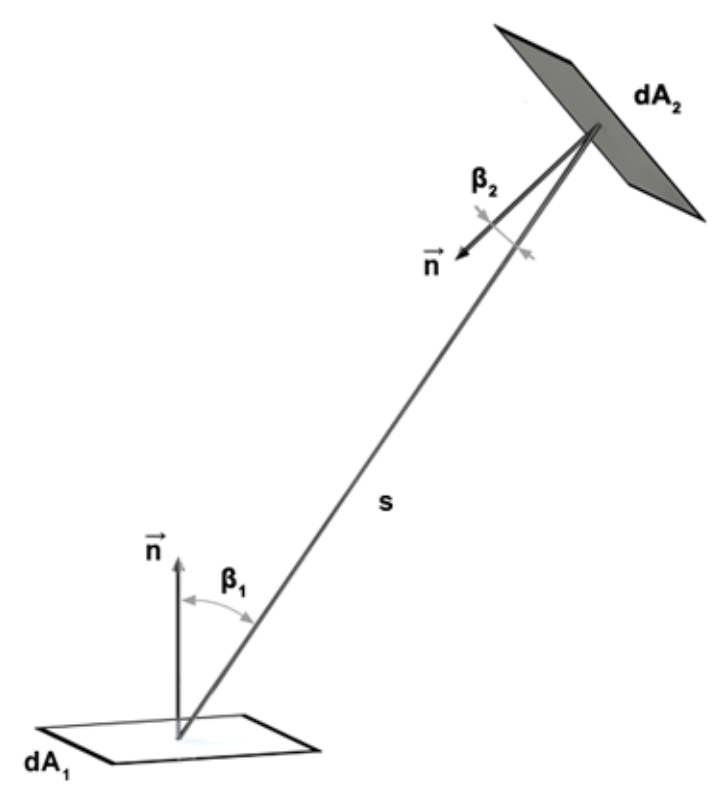

Figure 1. Sample configuration of two rectangular facets in $3 \mathrm{D}$.

direction of the outer normal due to radiation can be expressed as

$$
Q_{i}=\sum_{j=1}^{n} \frac{B_{i}-B_{j}}{1 / A_{i} F_{i-j}}, \quad i \neq j .
$$

The model presented here is designed to determine the heat flux distribution (5) all over any surface [8 10. Our model is composed of a set of particle surfaces, whereby each particle surface has given particular properties (location, size, emittance and temperature).

\section{Model implementation}

The proposed model was implemented into the IRE Designer computer program, which was written in Java language. IRE Designer implements our own numerical solver. The implementation worked with the $2 \mathrm{D}$ restriction of the system described in Section 2 . The application (Figure 2) deals with a cross-section of a heat emitter or emitters (with a reflector if needed) and the heated-up surface. The model geometry consists of a set of straight lines (line segments). In the $2 \mathrm{D}$ implementation, the view factors are evaluated by means of Hottel's Crossed Strings method [9]. The advantage of the two-dimensional model is its significant simplification and its straightforward view-factor evaluation, and it also responds promptly to input parameter changes. The aim of a particular simulation was to obtain the heat flux distribution all over the heated surface under given conditions that were in general given by the temperature and the material properties of particular line segments.

An additional functionality has been implemented in order to validate the evaluated results according to measured data (through the import of measured values). In addition, an algorithm to optimize the 


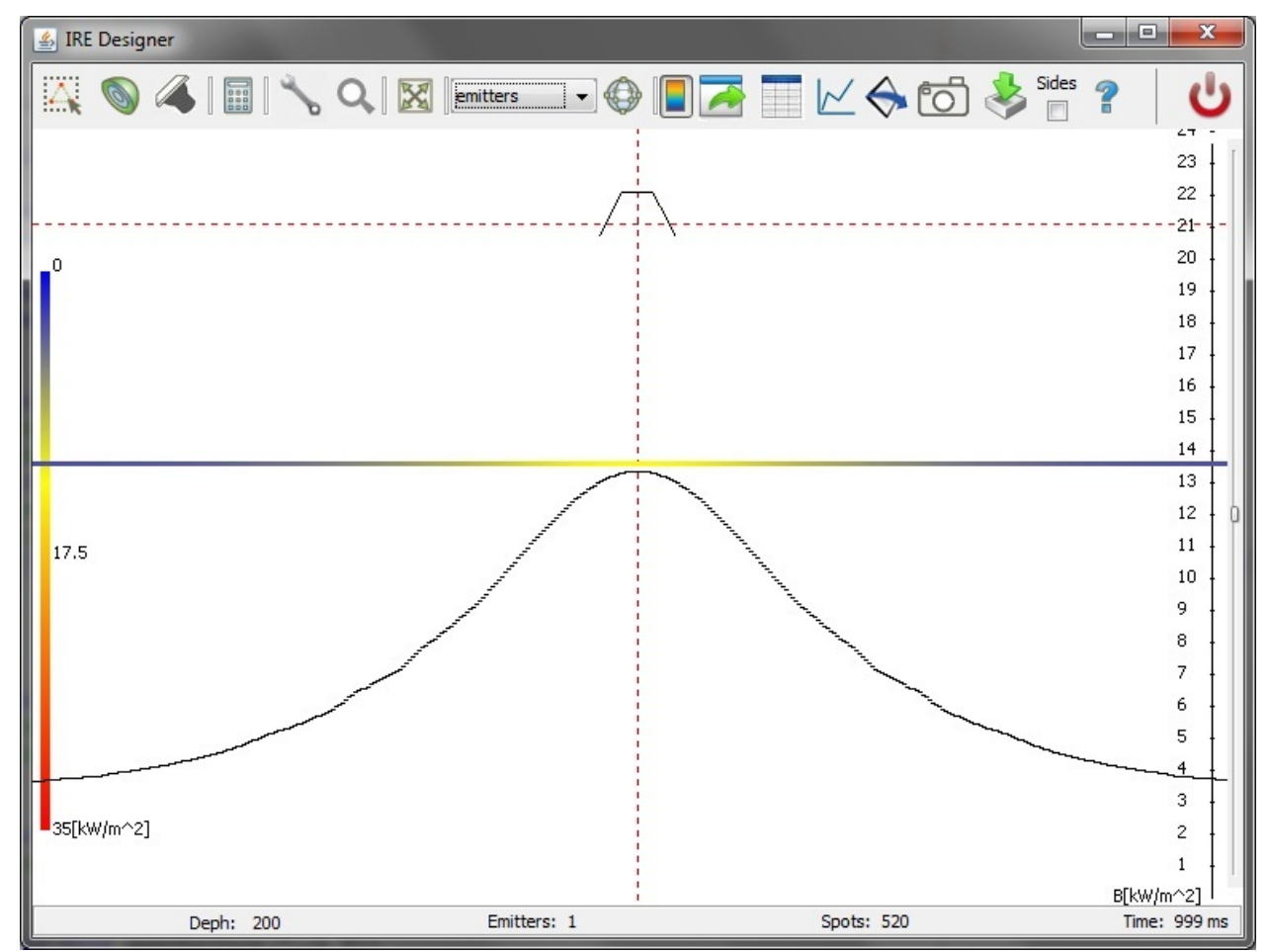

FIGURE 2. Illustrative screenshot of IRE Designer's main window with a simple simulation.

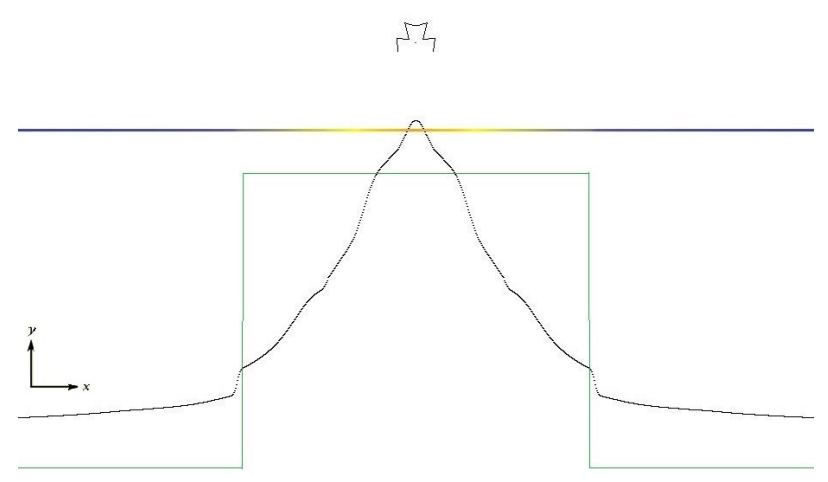

Figure 3. An intermediate result of incomplete reflector shape optimization. The figure shows the required heat flux distribution by the green rectangular line and the simulated distribution.

shape of a reflector to reach the desired heat flux distribution function has been implemented into the two-dimensional version of the model.

Reflector shape optimization uses the Hill-climbing algorithm, which is based on accomplishing small steps in the direction of the gradientt [1]. The intermediate result of an incomplete reflector shape optimization is illustrated in Figure 3. The optimization is carried out in subsequently-performed steps. In each step of the algorithm, the aim is to modify the position of each point that represented the reflector partitioning (boundary points of line segments forming the reflector).

The considered position modifications of one point are shown in Figure 4. When each point has been moved, the difference between the simulated and the

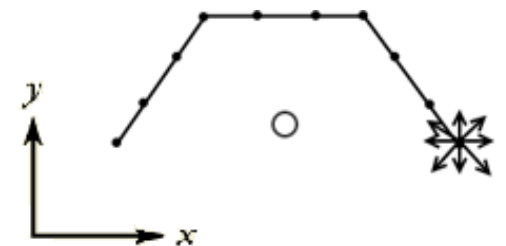

Figure 4. The considered shifts of a single point of the two dimensional reflector model used for each step in shape optimization.

desired heat intensity was evaluated. Those intensities are shown in Figure 3 The desired intensity distribution is distinguished by a green line. The second curve is for the calculated distribution of the particular configuration. The optimisation starts with a user-defined initial configuration and material and temperature properties. In the case of reflector shape optimisation presented in Figure 3, we consider flat heated surface. In that case, the following parameters were used: temperature of the heated surface $110^{\circ} \mathrm{C}$, temperature of the heater wire $3230^{\circ} \mathrm{C}$, and temerature of the reflector $300^{\circ} \mathrm{C}$; the emissivity of the heated surface 0.73 , emissivity of the heater wire 0.95 and emissivity of the reflector 0.05 . Initial values used for the optimization algorithm are determined by the model calibration described in Section 5. In each step of the algorithm, the best configuration is selected. The intermediate result was the outcome for the next optimization step. The optimization algorithm minimizes the difference between the absolute values of the integrals of the simulated and the desired distribution function. 

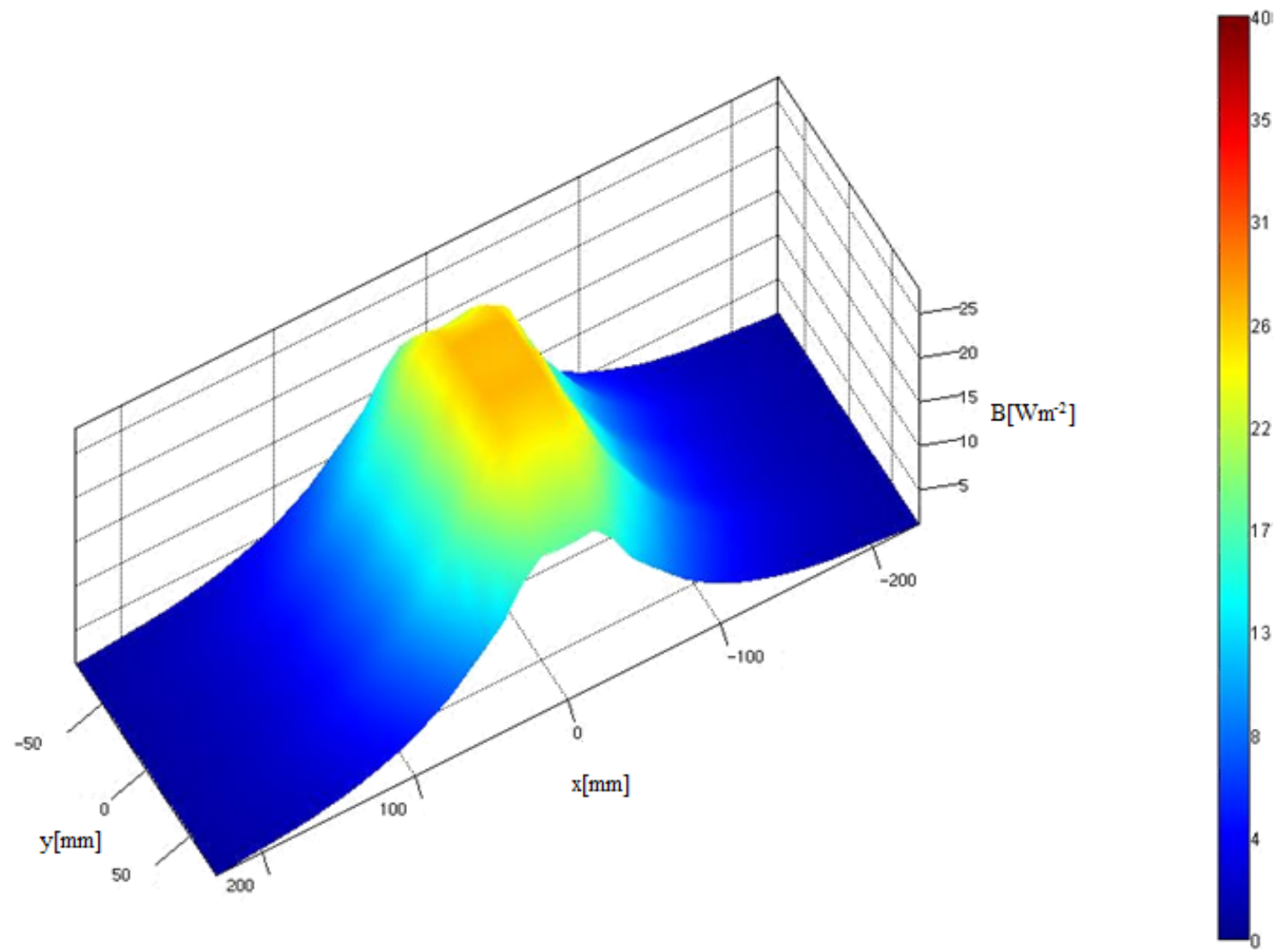

FigURE 6. 3D visualization of the measured heat flux characteristics of the Phillips IRZ500 emitter placed at distance of $100 \mathrm{~mm}$ above the heated surface.

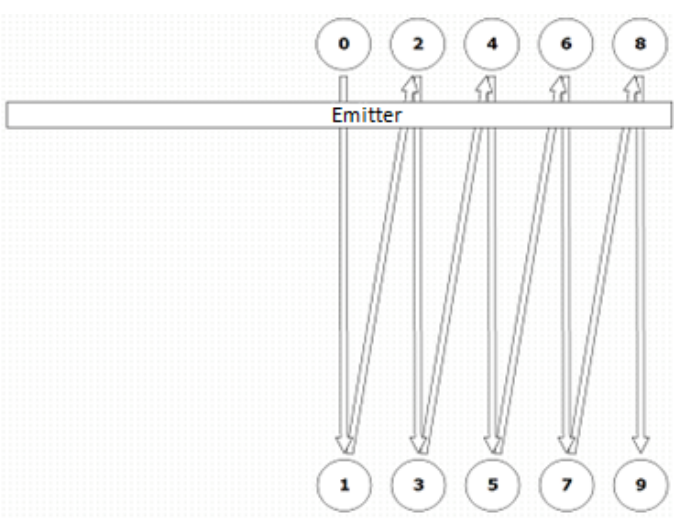

FiguRE 5. The trajectory of the heat flux characteristics of measured emitter.

\section{HeAt FLUX MEASUREMENT}

For validation of the simulated results against real results, we performed a heat flux measurement of a real infrared heater. The real measurement was made with a Hukseflux SBG01 heat flux sensor. The detected emissivity of the sensor was 0.95 , showing almost "black body" behaviour. Due to the symmetry, the measurement of the heat flux was performed in one quadrant of the radiated surface. The trajectory of the measurement is shown in Figure 5 . A 3D visualization of the measured heat flux characteristics of the Phillips IRZ500 emitter placed at a distance of $100 \mathrm{~mm}$ above the heated surface is shown in Fig-

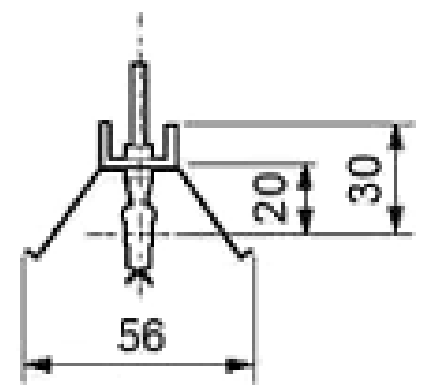

FiguRE 7. A side-view of the reflector used for measurements of the calibration values.

ure 6 The reflector that wasused is made of polished aluminum with spreadsheet emissivity values between 0.04 and 0.1 . The values shown in the graph were interpolated and mirrored on the other quadrants to achieve a 3D full visualization of the heat flux distribution. More information on the measurements, their conditions and results were presented in [12].

\section{MODEL CALiBRATiON}

The input data for a particular simulation performed on the given geometry (given the bounding surfaces and their discretization) are represented with the temperature and the emittance, for each particle surface (facet). For the temperature, setting a temperature of the heat emitter is the most crucial factor. The data provided in the documentation of particular products 


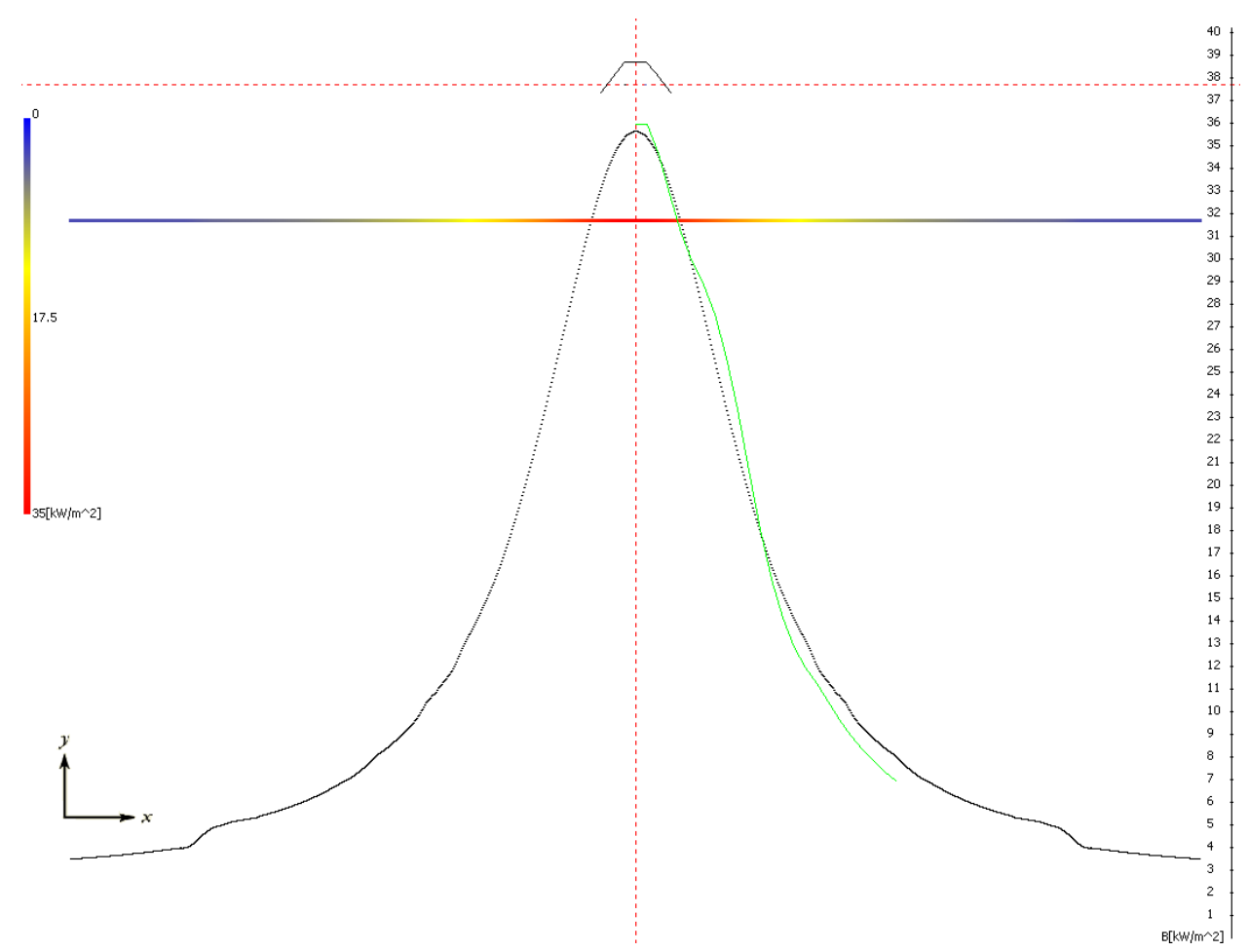

Figure 8. A validation of the measured and simulated values. The green line displays measured values and the black curve displays values obtained from the numerical simulation.

are not sufficient. However the results of our model were significantly influenced by that input. The heater temperature was calibrated with the Phillips IRZ500 emitter [13] based on results o real measurement. A side-view of the reflector used for this purpose is shown in Figure 7

Considering the three parts of the simulated system (the heater, the reflector, and the heated surface) and taking into account the uniform input characteristics of each of these parts, we obtained model inputs (as a result of our calibration): the temperature of the heated surface was $110^{\circ} \mathrm{C}$, the temperature of the heater wire was $3230{ }^{\circ} \mathrm{C}$, and the temperature of the reflector was $300^{\circ} \mathrm{C}$; the emissivity of the heated surface was 0.73 , the emissivity of the heater wire was 0.95 (an almost ideal black body surface), and the emissivity of the reflector was 0.05 . The calibration of all parameters was based on the table values for emissivity and the expected temperature of the elements. The calibration process is performed by minimizing the absolute value of the difference of the integrals of the simulated and measured distribution function.

A comparison of the measured and the simulated values of the calibrated numerical model is shown in Figure 8, where the green line on the right side represents the measured values, and the black curve represents the values obtained from the numerical simulation. Despite the calibration, it can be seen tat full agreement was not reached. The distinct course of the measured and simulated heat flux distribution is caused mainly by the simplification of the real system that is taken into accont in the model (e.g. the usage of a glass bulb filled with halogen). The main aim of the calibration is to determine the slope and the maximum of the heat flux distribution function.

\section{Model Results}

The main benefit of the implemented model is the possibility to obtain the heat flux characteristics in free-choice conditions given by the values of the temperature and the emissivity for each particular part of the model. A result of such a simulation is provided in Section 5. Here, the result was obtained through calibration based on validation by the measured data. The important feature of the model (with a view to its further use in real conditions) is its sensitivity to the input parameters. The impact of an inperfect/inprecise assessment of the inputs is determined by the sensitivity of the model. The graphs in Figure 9 and Figure 10 show the sensitivities to the temperature and the emissivity of various parts. The horizontal axisindicates deviation of the input parameters from the reference point given by the input parameters of the calibrated model described in Section 5 The vertical axisindicates the maximum heat flux under the reflector. Almost linear dependence can be seen in each of the outlined cases. In addition, for the input temperature settings, the results of the model were most sensitive to the heater temperature setting. However, the results were nearly independent from the reflector temperature, and for that reason we assume that its temperature can be set freely - (more or less) inaccurately — as its setting does not affect the results significantly. With regard to the input 


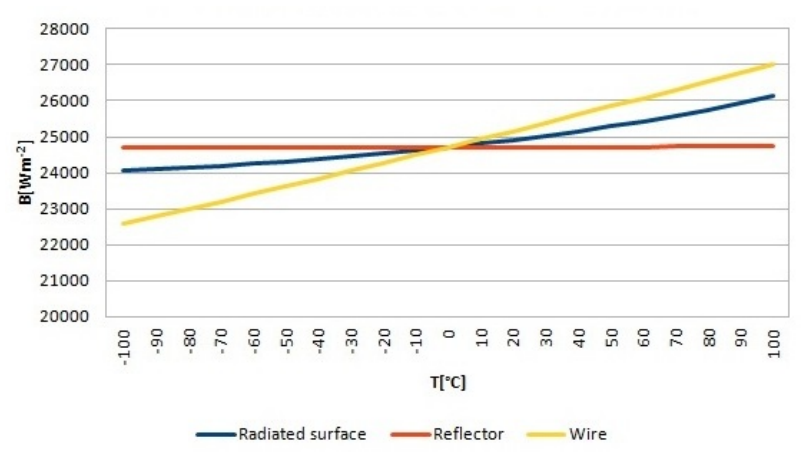

FIGURE 9. Model sensitivity to changes intemperature.

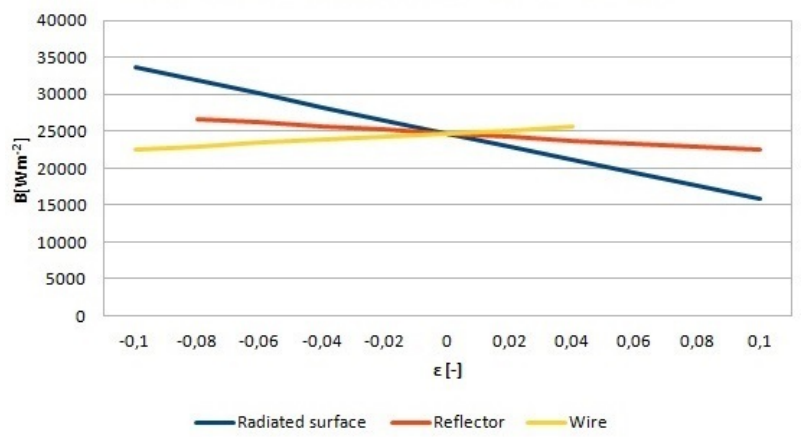

Figure 10. Model sensitivity to changes in emissivity.

emissivity settings, we observed that the results of the model were most sensitive to the radiated surface emissivity, whereas the emissivity of the heater wire and of the reflector had a less significant influence. With regard to the real system - heated-up shell-forms in the production of artificial leathers in the automotive industry - changes in the radiated surface quality are observed with the course of time, and for this reason the surface emissivity of the corresponding model must be adjusted appropriately.

In the model presented here, we have focused on a model involving a single heater. However, shell-forms in the production of artificial leathers are heated by tens (often more than one hundred) of infrared heaters. In the model of such a complex system, it is necessary to deal with the interaction of several heaters to determine the heat flux above a particular (defined) element of a heated form. We assume that a simple superposition (sum) of the particular contributions of heaters involved can be used in the calculations. Our presumption has been confirmed by preliminary measurements 1]. Then, we had to prove the applicability of the superposition in the procedure, using single heater model outputs for an evaluation of the complex model. For that reason, we dealt with a model involving more than one heater. Figure 11 shows a particular configuration of two heaters and their placement. For this case, the graph in Figure 12 shows comparison of (1) the heat flux obtained by summing the heat flux of two single heaters, and (2) the result obtained by the model with two infrared heaters. It can be seen, that there is a difference between the

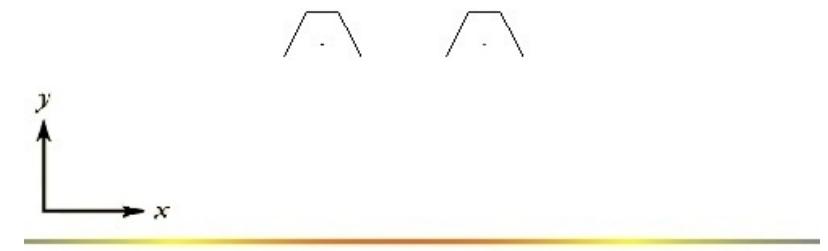

Figure 11. Two emitters side-by-side.

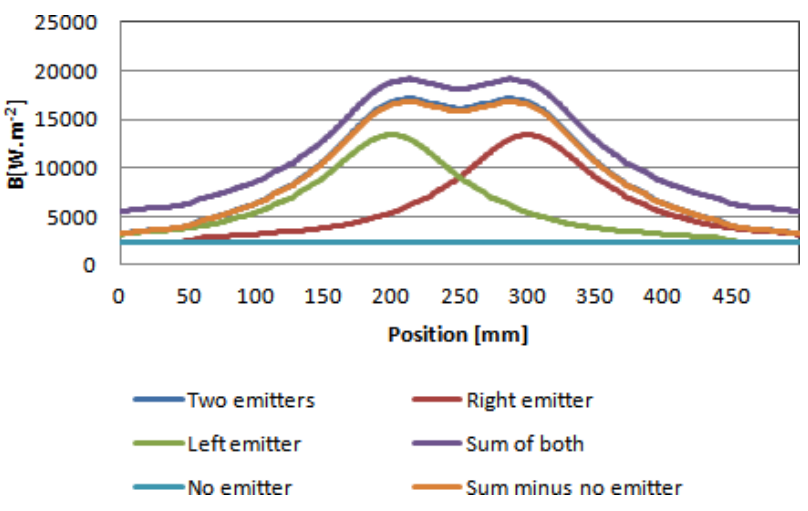

FIGURE 12. A comparison of heat flux intensity for each emitter, sum of flux of those emitters and simulated intensity of those emitters.

two curves. The difference represents the heat flux of the system involving only heated surface (without any heater nor reflector) - i.e. the heat flux of the surface emissivity, or the background heat flux. We had to avoid multiple involvement of the background heat flux when particular results were superposed in the single heater model.

The presented model is also helpful in solving task related to reflector design (shape), where its shape is determined by a desired heat flux distribution. A possible use and one example were mentioned in the Section 3 The model is also useful for determining the energy coverage in various configurations. Table 1 and Table 2 shows several configurations with different depths between the emitter and the heated surface. From those simulation results is possible to obtain sharpness of the radiation heat transfer characteristics for various situations. Table 2 presents the maximal value of the absorbed heat flux $B\left(\mathrm{Wm}^{-2}\right)$. Table 2 shows the amount of radiated energy $Q(\mathrm{~W})$ irradiating heated surfaces of different sizes, which indicates the fraction of the energy absorbed by surfaces of various widths.

\section{Conclusions}

This paper has presented a numerical model based on radiation heat transfer theory, and on utilization in simulations of a system consisting of an infrared heater, a heater reflector and the heated surface. The goal of a single simulation is to determine the heat flux distribution above the heated surface. The model presented here is the two-dimensional. Additionally, a super-structure module (using the model) applies an 


\begin{tabular}{lcccccc}
\hline Distance between surface and emitter $d$ & $50 \mathrm{~mm}$ & $10 \mathrm{~mm}$ & $150 \mathrm{~mm}$ & $200 \mathrm{~mm}$ & $250 \mathrm{~mm}$ & $300 \mathrm{~mm}$ \\
\hline Maximal heat flux $B$ & 72397 & 37255 & 25351 & 19463 & 15962 & 13644 \\
\hline
\end{tabular}

TABLE 1. Comparison of simulation results for emitters located at various depth above the heated surface. The table shows the maximal heat flux $B\left(\mathrm{~W} \mathrm{~m}^{-2}\right)$ for various depths.

\begin{tabular}{llllllcc}
\hline Distance between surface and emitter $d$ & $50 \mathrm{~mm}$ & $10 \mathrm{~mm}$ & $150 \mathrm{~mm}$ & $200 \mathrm{~mm}$ & $250 \mathrm{~mm}$ & $300 \mathrm{~mm}$ \\
\hline Heat transfer $Q$ for surface width $200 \mathrm{~mm}$ & 39709 & 38488 & 37728 & 36098 & 34425 & 32867 \\
Heat transfer $Q$ for surface width $160 \mathrm{~mm}$ & 38226 & 36914 & 35314 & 33213 & 31282 & 29405 \\
Heat transfer $Q$ for surface width $80 \mathrm{~mm}$ & 34756 & 30762 & 26854 & 23530 & 20842 & 18666 \\
Heat transfer $Q$ for surface width $40 \mathrm{~mm}$ & 30168 & 22689 & 17716 & 14454 & 12200 & 10578 \\
\hline
\end{tabular}

TABLE 2. A comparison of the simulation results for an emitter located at various depth above a heated surface. The table shows the rate of heat transfer $Q(\mathrm{~W})$ for heated surfaces of various widths.

optimization algorithm and serves to design a reflector (its shape). Both the numerical model and the superstructure optimizing module were implemented in the IRE Designer software tool. This tool is helpful in providing heat flux distribution functions under various configurations (heater reflector shape, heated surface shape and inclination, distance) and under various input parameters (temperature, emissivity).

The resulting heat flux distribution functions are used in the complex model of the heated shell-forms in the production of artificial leathers. Then, the optimization functionality is applicable for the design of a reflector utilizable above a particular part of the shell-form to avoid deep non-uniformity of the heat flux.

In addition, we are interested in an implementation corresponding to a full three-dimensional model, which has not been presented in this paper. Our future work is focused on implementing a more precise method that will determine the three-dimensional view factor with obstructions, and will be helpful for implementing a suitable generic optimization algorithm.

\section{ACKNOWLEDGEMENTS}

This work was supported by the Student Grant Competition of the Technical University of Liberec.

\section{REFERENCES}

[1] Martinec, T. Měreni teplot pomocí kontaktnich metod měrení. Ph.D. thesis, Technical university of Liberec, 2009.

[2] Školník, T. Řizeni teplotnich polí pomocí ohřevu infračervenými záriči. Ph.D. thesis, Technical university of Liberec, 2010.

[3] Mlýnek, J., Srb, R. Optimization of a heat radiation intensity on a mould surface in the car industry. In
Proceedings of 9th International Conference on Mechatronics. Springer-Verlag, Berlin, 2011.

[4] S. A. Rukolaine. The shape gradient of the least-squares objective functional in optimal shape design problems of radiative heat transfer. Journal of Quantitative Spectroscopy and Radiative Transfer 111(16):2390-2404, 2010. DOI:10.1016/j.jqsrt.2010.06.016.

[5] Hušek, M, Potěšil, A. Software prediction of non-stationary heating of shell moulds for manufacture of artificial leathers. In Proceedings of 18th International Conference ENGINEERING MECHANICS 2012. Svratka, Ždár nad Sázavou, 2012.

[6] K. M. Takami, Ö. Danielsson, J. Mahmoudi. High power reflector simulation to optimise electrical energy consumption and temperature profile. Applied Thermal Engineering 31(4):477-486, 2011. DOI:10.1016/j.applthermaleng.2010.09.031

[7] G. Walton. Calculation of Obstructed View Factors by Adaptive Integration. National Institute of Standards and Technology, 2002.

[8] F. M. Modest. Radiative heat transfer. 3rd ed. Academic Press, 2013.

[9] S. A. F. Hottel, H.C. Radiative Transfer. McGraw-Hill, New York, 1967.

[10] J. H. Lienhard IV, J. H. Lienhard V. A heat transfer textbook. 3rd ed. Phlogiston Press, 2003.

[11] A. W. Johnson, S. H. Jacobson. A class of convergent generalized hill climbing algorithms. Applied Mathematics and Computation 125:359-373, 2002. DOI:10.1016/S0096-3003(00)00137-5

[12] J. Mlýnek, A. Potěšil. Ohřevy radiací - teorie a průmyslová praxe. Technical university of Liberec, 2012.

[13] Dr. Fischer Europe S.A.S. Infrared halogen lamps, 2013. [2014-12-01], http://www.dr-fischer-group. com/img_pool/DF\%20IR\%20Catalogue.pdf 